\title{
Expression profile of class I histone deacetylases in human cancer tissues
}

\author{
MASAMUNE NAKAGAWA ${ }^{1}$, YOSHINAO ODA ${ }^{1}$, TAKASHI EGUCHI ${ }^{1}$, SHIN-ICHI AISHIMA ${ }^{1}$, \\ TAKASHI YAO ${ }^{1}$, FUMIHITO HOSOI ${ }^{3,5}$, YUJI BASAKI ${ }^{3}$, MAYUMI ONO ${ }^{3,4}$, \\ MICHIHIKO KUWANO ${ }^{5}$, MASAO TANAKA ${ }^{2}$ and MASAZUMI TSUNEYOSHI ${ }^{1}$

\begin{abstract}
Departments of ${ }^{1}$ Anatomic Pathology, ${ }^{2}$ Surgery and Oncology, ${ }^{3}$ Medical Biochemistry, ${ }^{4}$ Station-II for Collaborative Research, Graduate school of Medical Sciences, Kyushu University, 3-1-1 Maidashi, Higashi-ku, Fukuoka, 812-8582; ${ }^{5}$ Research Center for Innovative Cancer Therapy, Kurume University School of Medicine, 67 Asahimachi, Kurume 830-0011, Japan
\end{abstract}

Received March 23, 2007; Accepted June 19, 2007

\begin{abstract}
Histone deacetylase (HDAC) activity is one of the widely used and well-established mechanisms for regulation of various genes in cancer. To identify which subtype of class I HDACs are overexpressed in cancers, we analyzed the expression of class I HDAC isotypes composed of HDAC1, 2, 3 and 8 in several cell lines and human cancer tissues, including cancer of the stomach, esophagus, colon, prostate, breast, ovary, lung, pancreas and thyroid. The results showed that $>75 \%$ of human cancer tissues and their corresponding non-cancerous epithelium showed high expression of these class I HDACs. However, the immunoreactivity of HDAC8 in both prostatic cancer tissue and non-cancerous prostate glands was lower than that in other cancer tissues. Furthermore, $5-40 \%$ of cancer tissues overexpressed class I HDACs, when compared with normal epithelium. The results suggest the potential usefulness of HDAC inhibitors for the treatment of a wide variety of human cancers.
\end{abstract}

\section{Introduction}

Histone acetylation-deacetylation is considered to be the best understood of the post-translational modifications of the core histones, and occurs by the opposing action of histone acetylases (HATs) and histone deacetylases (HDACs) (1-7). To date, 18 HDAC enzymes have been identified in humans and divided into three subclasses based on amino acid sequence homology in their catalytic domains (8). Class I

Correspondence to: Dr Yoshinao Oda, Department of Anatomic Pathology, Graduate School of Medical Sciences, Kyushu University, 3-1-1 Maidashi, Higashi-ku, Fukuoka 812-8582, Japan

E-mail: oda@surgpath.med.kyushu-u.ac.jp

Key words: histone deacetylase, human cancer tissue, immunohistochemistry enzymes, which include HDACs 1, 2, 3 and 8, are related to yeast reduced potassium dependency 3 deacetylase and share homology in their catalytic sites $(8,9)$. Class I HDACs play an important role in the regulation of cell proliferation (10) and are expressed almost exclusively in the nucleus of most cell types $(8,11)$.

Overexpression of class I HDACs have been reported in several cancer tissues, such as stomach (12-14), esophagus $(15)$, colorectal $(16,17)$, prostate $(18,19)$, breast $(21)$ and lung $(22,23)$. However, systematic analysis of expression profiles of class I HDACs in various human cancer tissues has not yet been documented.

Currently, several HDAC inhibitors are in clinical trials, and are showing promising anticancer effects in phase I and II trials. To identify the HDAC inhibitors with specificity for cancer types and for specific HDAC classes or isoforms is of interest, and it is important to consider that HDACs have varying expression patterns in different cell types, as well as diverse functions (24).

To investigate expression of class I HDACs in cancer tissues, we first confirmed the expression profiles of class I HDACs in 11 major cancer cell lines. Next, we evaluated immunohistochemical nuclear staining of class I HDACs in nine types of carcinoma, including stomach, esophagus, colon, prostate, breast, ovary, lung, pancreas and thyroid.

\section{Materials and methods}

Cell lines and Western blot analysis. The following human cancer cell lines were purchased from the American Type Culture Collection (ATCC) (Manassas, VA, USA): lung (A549), breast (MCF-7 and MDA-MB-231), ovary (SKOV3), prostate (LNCap, PC-3 and DU145), pancreas (MiaPaCa-2 and PANC-1) and colon (SW480 and SW620). All cell lines were cultured in medium recommended by ATCC, supplemented with $10 \%$ fetal bovine serum under standard cell culture condition at $37^{\circ} \mathrm{C}$ and $5 \% \mathrm{CO}_{2}$ in a humid environment. Cells were rinsed with ice-cold PBS and lysed in buffer containing $50 \mathrm{mmol} / \mathrm{l}$ Tris/ $\mathrm{HCl}, 350 \mathrm{mmol} / 1 \mathrm{NaCl}, 0.1 \% \mathrm{NP} 40$, $5 \mathrm{mmol} / \mathrm{l} \mathrm{EDTA}, 50 \mathrm{mmol} / \mathrm{l} \mathrm{NaF}, 1 \mathrm{mmol} / \mathrm{l}$ phenylmethyl- 
sulfonyl fluoride, $10 \mu \mathrm{g} / \mathrm{ml}$ aprotinin, $10 \mu \mathrm{g} / \mathrm{ml}$ leupeptin, and $1 \mathrm{mmol} / 1 \mathrm{Na}_{3} \mathrm{VO}_{4}$. Cell lysates were subjected to SDSPAGE and blotted onto Immobilon membranes (Millipore, Bedford, MA, USA), as described previously (25). After transfer, blots were incubated with the blocking solution and probed with primary polyclonal antibodies, including antiHDAC1 antibody (1:25 dilution; Cell Signaling Technology, Danvers, MA, USA), anti-HADC2 antibody (1:1250 dilution; Affinity BioReagents, Golden, CO, USA), anti-HDAC3 antibody (1:500 dilution; Sigma, St. Louis, MO, USA), antiHDAC8 antibody (1:100 dilution; Santa Cruz Biotechnology, Santa Cruz, CA, USA) and anti- $\beta$-actin antibody (1:5000 dilution; Abcam, Cambridge, MA, USA). The protein content was visualized using horseradish peroxidase-conjugated secondary antibodies followed by enhanced chemiluminescence (Amersham, Piscataway, NJ, USA).

Clinical specimens and immunohistochemistry. Surgical specimens from advanced cancer of the stomach, esophagus, colon, prostate, breast, ovaries, lung, pancreas and thyroid were retrieved from the files of the Department of Anatomic Pathology, Kyushu University, Japan, for the period 19952005. For each of the nine types of carcinoma, 20 cases were selected from representative formalin-fixed paraffin-embedded materials. Consequently, 180 paraffin-embedded blocks were prepared for immunohistochemistry. Histological characteristics of each type of carcinoma are summarized in Table I. The specimens were immersed in $10 \%$ neutral-buffered formalin immediately after surgery, and embedded in paraffin blocks. Blocks containing both carcinoma and the corresponding normal epithelium were chosen for stomach, esophagus, colon and prostate cancer. After deparaffinization of $4-\mu$ m-thick sections, inhibition of endogenous peroxidase activity was achieved with methanol containing $3 \% \mathrm{H}_{2} \mathrm{O}_{2}$. Tissue sections were boiled in $0.1 \mathrm{M}$ sodium citrate buffer (pH 6.0) in a pressure cooker inside a microwave oven for $40 \mathrm{~min}$, to retrieve the antigen. The slides were incubated at $4^{\circ} \mathrm{C}$ overnight, with the same primary polyclonal antibodies as used in Western blotting. The working dilutions were as follows: HDAC1 (1:25), HDAC2 (1:1250), HDAC3 (1:500) and HDAC8 (1:100). The above procedures were followed by staining with a streptavidin/biotin/peroxidase kit (Nichirei, Tokyo, Japan). The sections were then reacted with 3,3diaminobenzidine as a chromogen, and counterstained with hematoxylin. The localization of HDAC1 and HDAC2 is exclusively nuclear (26). Although HDAC3 (27) and HDAC8 (28) are mainly localized in the nucleus, but can also localize in the cytoplasm, we evaluated only nuclear immunostaining.

Immunohistochemical evaluation of class I HDACs was subtle, because some degree of heterogeneity of class I HDAC expression was detected, even within some morphologically similar areas of the tumor, as previously described in gastric cancer tissues (13). Furthermore, there were no significant differences in nuclear staining intensity between cancer tissues and the corresponding normal epithelium in any of the nine types of tumor. We evaluated the extent of nuclear staining on a four-tiered scale $(1+, 0-25 \% ; 2+, 26-50 \% ; 3+, 51-75 \%$; $4+, 76-100 \%)$. The staining score was evaluated in 10 fields by low-power magnification, and only one stained spot was examined at high power $(\mathrm{x} 400)$. Each case was scored
Table I. Histopathological characteristics of several carcinomas.

Stomach

Cases

Well-differentiated adenocarcinoma

Moderately differentiated adenocarcinoma

Poorly differentiated adenocarcinoma

Esophagus

Well-differentiated squamous cell carcinoma

Moderately differentiated squamous cell carcinoma

Colon

Well-differentiated adenocarcinoma

Moderately differentiated adenocarcinoma

Prostate

Well-differentiated adenocarcinoma

Moderately differentiated adenocarcinoma

Poorly differentiated adenocarcinoma

Breast

Papillotubular carcinoma 7

Solid tubular carcinoma

Scirrhous carcinoma

Ovary

Clear cell adenocarcinoma 3

Mucinous adenocarcinoma 3

Endometrial adenocarcinoma 3

Serous adenocarcinoma

Lung

Squamous cell carinoma

Adenocarcinoma

Pancreas

Well-differentiated adenocarcinoma

Moderately differentiated adenocarcinoma

Poorly differentiated adenocarcinoma

Undifferentiated carcinoma

Thyroid

Papillary carcinoma

Breast tumors were classified according to the general rules for clinical and phathological records for breast cancer in Japan.

independently by three pathologists (M.N., O.Y. and S.A.). Samples with a score of $3+$ or $4+$ were defined as 'HDACpositive'. For negative controls, the slides were treated with non-immune serum rather than primary antibody.

\section{Results}

Western blot analysis. All class I HDACs, including HDAC1, 2, 3 and 8, were ubiquitously expressed in all 11 cancer cell lines (Fig. 1). This feature indicates that class I HDACs are expressed in many cancer tissues. Class I HDACs were 


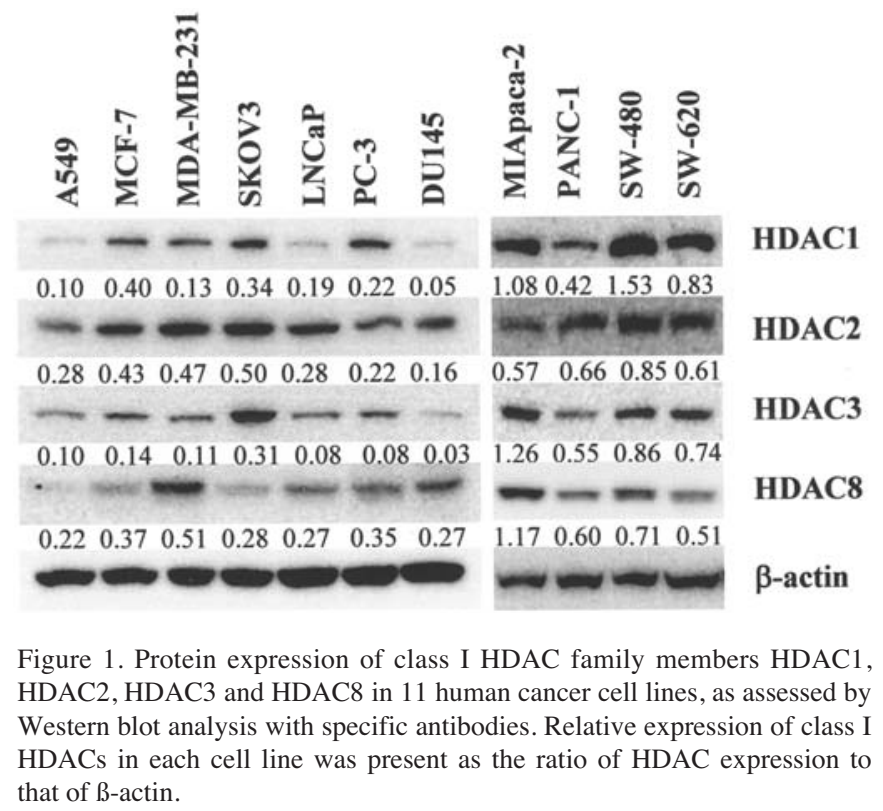

Table II. The number and rate of 'HDAC-positive' cases in each 20 examined carcinomas.

\begin{tabular}{|c|c|c|c|c|}
\hline Cancers & HDAC1 & HDAC2 & HDAC3 & HDAC8 \\
\hline \multicolumn{5}{|l|}{ Stomach } \\
\hline $\mathrm{C}$ & 17 (85) & 20 (100) & $20(100)$ & 19 (95) \\
\hline $\mathrm{N}$ & $17 \quad(85)$ & $20(100)$ & $17 \quad(85)$ & $15 \quad(75)$ \\
\hline \multicolumn{5}{|c|}{ Esophagus } \\
\hline $\mathrm{C}$ & 19 (95) & $20(100)$ & $19 \quad(95)$ & $18 \quad(90)$ \\
\hline $\mathrm{N}$ & $20(100)$ & 19 (95) & $16(80)$ & 15 (75) \\
\hline \multicolumn{5}{|l|}{ Colon } \\
\hline $\mathrm{C}$ & 19 (95) & $20(100)$ & $19 \quad(95)$ & $17 \quad(85)$ \\
\hline $\mathrm{N}$ & $18 \quad(90)$ & $20(100)$ & $19 \quad(95)$ & $18 \quad(90)$ \\
\hline \multicolumn{5}{|l|}{ Prostate } \\
\hline $\mathrm{C}$ & $15 \quad(75)$ & $20(100)$ & $20(100)$ & 7 (35) \\
\hline $\mathrm{N}$ & 17 & $17 \quad(85)$ & $19 \quad(95)$ & $6 \quad(30)$ \\
\hline Breast & $17 \quad(85)$ & $20(100)$ & $20(100)$ & $17 \quad(85)$ \\
\hline Ovary & 19 (95) & 20 (100) & $16(80)$ & $16(80)$ \\
\hline Lung & $20(100)$ & $20(100)$ & $20(100)$ & $17 \quad(85)$ \\
\hline Pancreas & 17 (85) & $18 \quad(90)$ & $20(100)$ & $18 \quad(90)$ \\
\hline Thyroid & $20(100)$ & $20(100)$ & $20(100)$ & $20(100)$ \\
\hline
\end{tabular}

Percentage values shown in parentheses; $\mathrm{C}$, cancer tissue; N, noncancerous tissue.

overexpressed in MIApaca-2, SW-480 and SW-620 cancer cell lines, compared with the other cancer cell lines.

Immunohistochemistry. The number and rate of each 'HDAC-positive' case in each cancer tissue are summarized
Table III. Comparison of the proportion score of class I HDACs in cancerous and non-cancerous tissues from four types of cancer.

\begin{tabular}{lrrrr}
\hline Cancers & HDAC1 & HDAC2 & HDAC3 & HDAC8 \\
\hline Stomach & & & & \\
$\mathrm{C}>\mathrm{N}$ & $5(25)$ & $1(5)$ & $6(30)$ & $5(25)$ \\
$\mathrm{C}=\mathrm{N}$ & $9(45)$ & $16(80)$ & $14(70)$ & $14(70)$ \\
$\mathrm{C}<\mathrm{N}$ & $6(30)$ & $3(15)$ & $0(0)$ & $1(5)$ \\
Esophagus & & & & \\
$\mathrm{C}>\mathrm{N}$ & $3(15)$ & $5(25)$ & $7(35)$ & $6(30)$ \\
$\mathrm{C}=\mathrm{N}$ & $10(50)$ & $11(55)$ & $8(40)$ & $10(50)$ \\
$\mathrm{C}<\mathrm{N}$ & $7(35)$ & $4(20)$ & $5(25)$ & $4(20)$ \\
Colon & & & & \\
$\mathrm{C}>\mathrm{N}$ & $2(10)$ & $0(0)$ & $1(5)$ & $4(20)$ \\
$\mathrm{C}=\mathrm{N}$ & $13(65)$ & $19(95)$ & $11(55)$ & $12(60)$ \\
$\mathrm{C}<\mathrm{N}$ & $5(25)$ & $1(5)$ & $8(40)$ & $4(20)$ \\
Prostate & & & & \\
$\mathrm{C}>\mathrm{N}$ & $6(30)$ & $8(40)$ & $7(35)$ & $7(35)$ \\
$\mathrm{C}=\mathrm{N}$ & $7(35)$ & $12(60)$ & $13(65)$ & $10(50)$ \\
$\mathrm{C}<\mathrm{N}$ & $7(35)$ & $0(0)$ & $0(0)$ & $3(15)$ \\
\hline
\end{tabular}

Percentage values shown in parentheses; $\mathrm{C}$, cancer tissue; $\mathrm{N}$, noncancerous tissue. Class I HDAC expression was compared between cancer tissues and normal epithelium. $\mathrm{C}>\mathrm{N}, \mathrm{C}=\mathrm{N}, \mathrm{C}<\mathrm{N}$ mean that class I HDAC expression in the cancer cells was more, equal to or less than that in the normal epithelial cells, respectively.

in Table II. The score for class I HDAC expression in the cancer tissues and normal epithelium was almost equal in four types of cancer, including stomach, esophagus, colon and prostate, therefore, expression of class I HDACs in four types of cancer tissues were compared with that in normal epithelium (Table III). Immunohistochemical findings in five types of human cancer and four types of normal epithelium are shown in Fig. 2.

Cancer and non-cancerous epithelium of the stomach. Immunoreactivity showed expression for HDAC1, HDAC2, HDAC3 and HDAC8 in 17 (85\%), 20 (100\%), 20 (100\%) and $19(95 \%)$ of 20 cases of gastric cancer, respectively (Fig. 2A). In addition, HDAC1, HDAC2, HDAC3 and HDAC8 were highly expressed in 17 (85\%), 20 (100\%), 17 $(85 \%)$ and $15(75 \%)$ of 20 corresponding samples of noncancerous gastric mucosa, respectively (Fig. 2B). HDAC1, HDAC2, HDAC3 and HDAC8 was overexpressed more in the cancer tissues than the normal epithelium in $5(25 \%), 1$ $(5 \%), 6(30 \%)$ and $5(25 \%)$ cases, respectively (Table III).

Cancer and non-cancerous epithelium of the esophagus. Immunoreactivity for HDAC1, HDAC2, HDAC3 and HDAC8 was observed in 19 (95\%), 20 (100\%), 19 (95\%) and 18 (90\%) of 20 cases of esophageal cancer, respectively (Fig. 2C). 
HDAC1

A, SC, 1-4

B, NS, 1-4

C, EC, 1-4

D, NE, 1-4

E, PC, 1-4

F, NP, 1-4
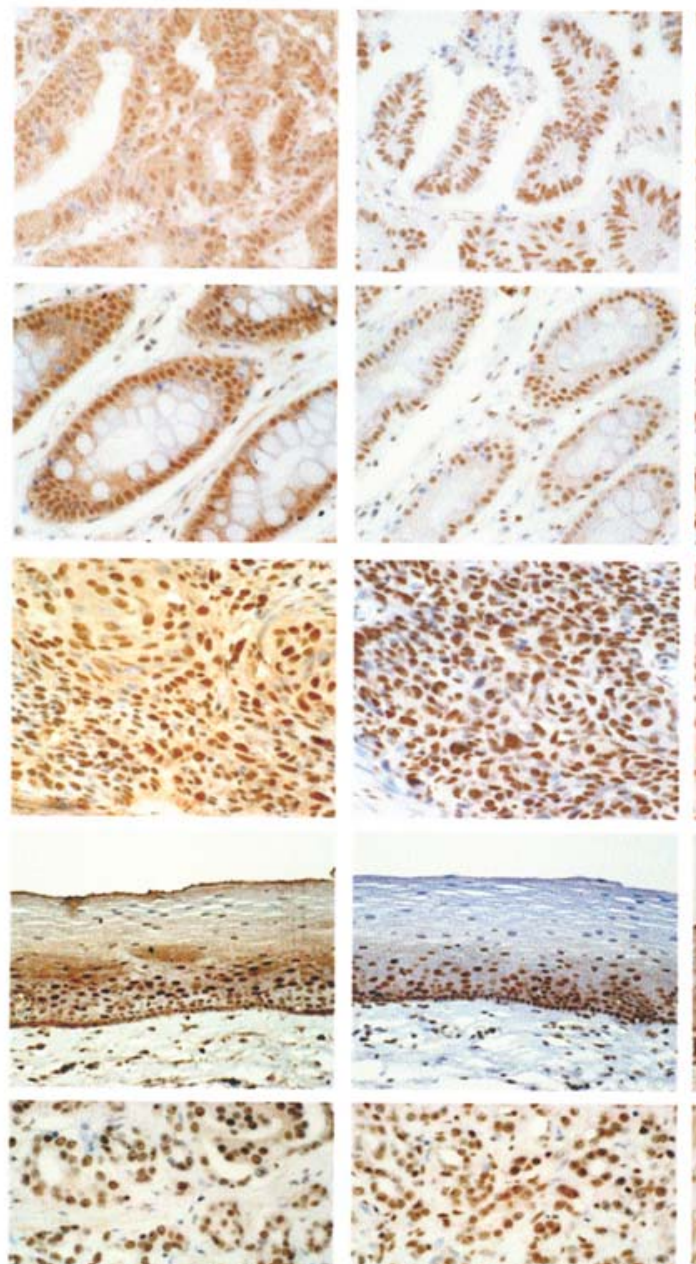

HDAC3
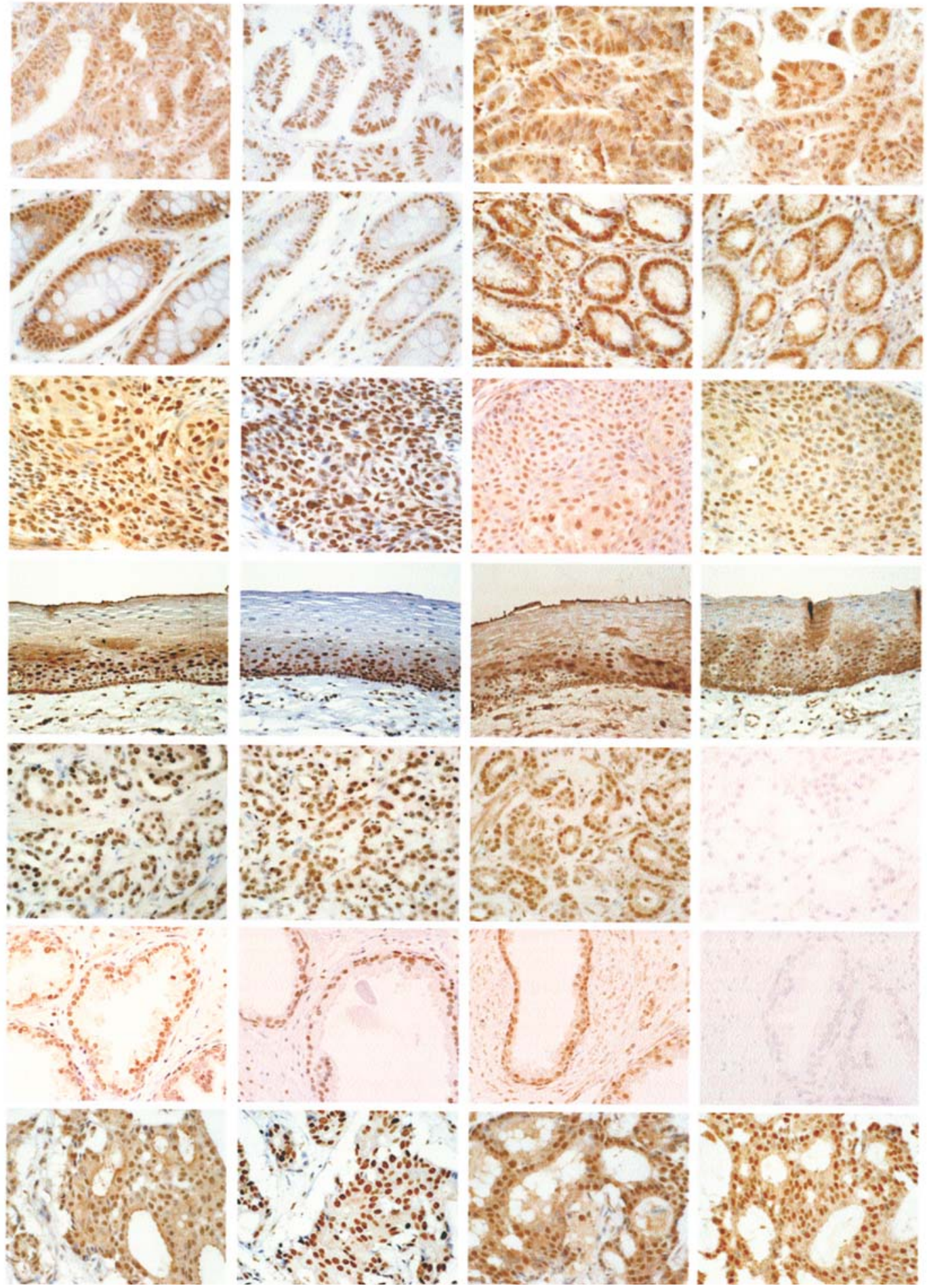

Figure 2. Immunohistochemistry of class I HDAC expression in several types of human cancer tissues and corresponding non-cancerous epithelium. (SC, stomach carcinoma; NS, normal stomach epithelium; EC, esophagus carcinoma; NE, normal esophagus epithelium; PC, prostate carcinoma; NP, normal prostate epithelium; BC, breast carcinoma) (A). Immunostaining of well-differentiated gastric adenocarcinoma with HDAC1 (A1), HDAC2 (A2), HDAC3 (A3) and HDAC8 (A4), showing strong nuclear expression. Cytoplasmic staining was also recognized for HDAC8. (B) Immunostaining of gastric non-cancerous epithelium with HDAC1 (B1), HDAC2 (B2), HDAC3 (B3) and HDAC8 (B4), showing strong nuclear expression. (C) Immunostaining of well-differentiated esophageal squamous cell carcinoma with HDAC1 (C1), HDAC2 (C2), HDAC3 (C3) and HDAC8 (C4), showing strong nuclear expression. Cytoplasmic staining was also recognized for HDAC8. (D) Immunostaining of non-cancerous esophageal epithelium with HDAC1 (D1), HDAC2 (D2), HDAC3 (D3) and HDAC8 (D4), showing strong nuclear expression. The expression of class I HDACs was mainly localized at the basal layer, whereas no immunostaining was observed in the keratinizing superficial layer. (E) Immunostaining of well-differentiated prostate adenocarcinoma with HDAC1 (E1), HDAC2 (E2), HDAC3 (E3) and HDAC8 (E4), showing strong nuclear expression. Cytoplasmic staining was also recognized for HDAC8. Immunoreactivity for HDAC 8 was lower than that in other cancer tissues. (F) Immunostaining of non-cancerous prostate epithelium with HDAC1 (F1), HDAC2 (F2), HDAC3 (F3) and HDAC8 (F4), showing strong nuclear expression. Immunoreactivity for HDAC 8 was lower than that in other normal epithelium. (G) Immunostaining of breast papillotubular carcinoma with HDAC1 (G1), HDAC2 (G2), HDAC3 (G3) and HDAC8 (G4), showing strong nuclear expression. Cytoplasmic staining was also recognized for HDAC8. 
HDAC1, HDAC2, HDAC3 and HDAC8 was immunopositive in $20(100 \%), 19(95 \%), 16(80 \%)$ and $15(75 \%)$ of 20 samples of corresponding non-cancerous squamous epithelium of the esophagus, respectively (Fig. 2D). In the corresponding non-cancerous esophageal epithelium, the expression of class I HDACs was mainly localized at the basal and intermediate layers, whereas no immunostaining was observed in keratinizing superficial layers (Fig. 2D). HDAC1, HDAC2, HDAC3 and HDAC8 were overexpressed more in the cancer tissues than the normal epithelium in $3(15 \%), 5$ (25\%), 7 (35\%) and 6 (30\%) cases, respectively (Table III).

Cancer and non-cancerous epithelium of the colon. Immunoreactivity for HDAC1, HDAC2, HDAC3 and HDAC8 was observed in 19 (95\%), 20 (100\%), 19 (95\%) and $17(85 \%)$ of 20 cases of colon cancer, respectively. HDAC 1, HDAC2, HDAC3 and HDAC8 was positive in 18 (90\%), 20 (100\%), $19(95 \%)$ and $18(90 \%)$ of 20 specimens of corresponding non-cancerous colon mucosa, respectively. HDAC1, HDAC2, HDAC3 and HDAC8 were overexpressed more in cancer tissues than in normal colon epithelium in $2(10 \%), 0(0 \%), 1$ $(5 \%)$ and 4 (20\%) cases, respectively (Table III).

Cancer and non-cancerous epithelium of the prostate. Immunoreactivity for HDAC1, HDAC2, HDAC3 and HDAC8 was observed in $15(75 \%), 20(100 \%), 20(100 \%)$ and 7 (35\%) of 20 cases of prostate cancer, respectively (Fig. 2E). HDAC1, HDAC2, HDAC3 and HDAC8 was positive in 17 $(85 \%), 17(85 \%), 19(95 \%)$ and $6(30 \%)$ of 20 specimens of non-neoplastic prostate, respectively (Fig. 2F). Both the epithelial and myoepithelial cells of the prostate equally expressed these class I HDACs. Immunoreactivity for HDAC8 in both cancer tissue $(35 \%)$ and non-cancerous prostate $(30 \%)$ was lower than that in other cancer tissues. Generally, overexpression of class I HDACs is observed in hyperplastic glands, whereas atrophic glands show reduced expression. HDAC1, HDAC2, HDAC3 and HDAC8 was overexpressed more in the cancer tissues than the normal epithelium in $6(30 \%), 8(40 \%), 7(35 \%)$ and $7(35 \%)$ cases, respectively (Table III).

Breast cancer. Immunoreactivity for HDAC1, HDAC2, HDAC3 and HDAC8 was positive in 17 (85\%), 20 (100\%), $20(100 \%)$ and $17(85 \%)$ of 20 cases of breast cancer, respectively (Fig. 2G).

Ovarian cancer. Immunoreactivity for HDAC1, HDAC2, HDAC3 and HDAC8 was observed in 19 (95\%), 20 (100\%), $16(80 \%)$ and $16(80 \%)$ of 20 cases of ovarian cancer, respectively. There was no significant difference in the expression profiles between histological subtypes.

Lung cancer. Immunoreactivity for HDAC1, HDAC2, HDAC3 and HDAC8 was observed in 20 (100\%), 20 (100\%), $20(100 \%)$ and $17(85 \%)$ of 20 cases of lung cancer, respectively. No significant difference in immunoreactivity was observed between adenocarcinoma and squamous cell carcinoma.

Pancreas cancer. Immunoreactivity for HDAC1, HDAC2, HDAC3 amd HDAC8 was observed in 17 (85\%), 18 (90\%),
$20(100 \%)$ and $18(90 \%)$ of 20 cases of pancreas cancer, respectively.

Thyroid cancer. Immunoreactivity for HDAC1, HDAC2, HDAC3 and HDAC8 was observed in 20 (100\%), 20 (100\%), $20(100 \%)$ and $20(100 \%)$ of 20 cases of thyroid cancer, respectively. Twenty cases of thyroid papillary carcinoma were all immunoreactive for class I HDACs.

\section{Discussion}

There have been several reports that the class I HDACs are upregulated in many cancer cell lines and tissues, at both the transcriptional and translational levels. However, systematic analysis of the expression profiles of class I HDACs in human cancer tissues has not been carried out. Overexpression of class I HDACs have been reported in several cancer tissues $(12-23)$. In gastric cancer, overexpression of HDAC1 $(13,14)$ and HDAC2 (12) has been investigated and 76\% (38/50) of advanced gastric cancer shows a high rate of HDAC2 expression (12). In breast cancer, overexpression of HDAC1 has been observed in $39.8 \%(70 / 176)$, and HDAC3 in $43.9 \%$ $(76 / 173)$ of patients (21). In lung cancer, 62\% (28/45) of cancer tissues were immunohistochemically stained for HDAC1 in more than $10 \%$ of tumor cells (22). Halkidou et al have assessed HDAC1 immunostaining in prostate cancer, with both a proportion and intensity score (20). In their study, $100 \%$ of the hormone-refractory (14/14) and low-grade prostate $(5 / 5)$ cancer showed moderate to strong nuclear expression, and benign prostatic hyperplasia showed weak nuclear expression. As for colon cancer, Zhu et al (16) have shown that $82 \%(47 / 57)$ of colon cancer patients overexpress HDAC2.

To evaluate the expression of class I HDAC proteins in several types of human cancer, we first analyzed the expression of class I HDAC isotypes consisting of HDAC1, 2, 3 and 8 in 11 cell lines from six different types of cancer, by Western blotting. Class I HDACs are ubiquitously expressed in many cancer cell lines, and the expression level of class I HDACs seems to be different in each cancer tissue and each class I HDAC.

We next investigated class I HDAC proteins by immunohistochemistry. Although most previous studies have evaluated class I HDACs by intensity of nuclear staining, we evaluated the expression in cancer tissues by the proportion of nuclei stained, because class I HDAC nuclear staining intensity was strong in both cancer tissues and corresponding normal epithelium. In the current study, some degree of heterogeneity of class I HDAC staining was detected in our nine types of cancer tissue, as previously described in gastric cancer tissues (13). Therefore, we employed conventional evaluation of grossly mounted and immunostained slides, instead of the tissue microarray method.

In the current study, class I HDACs were highly expressed in tissues from stomach, esophagus, colon, breast, ovaries, lung, pancreas and thyroid cancer, but expression of HDAC8 in prostate cancer was definitely lower than that in the other cancer tissues. Waltregny et al found that HDAC8 immunoreactivity was not detected in either normal or malignant epithelial cells in any of 24 prostate cancer tissues (19). 
Considering these results, there is a possibility that HDAC8 expression levels were decreased more in prostate cancer than in other cancers, but the reason for this remains unknown.

In our study, $5-40 \%$ of cancer tissues overexpressed class I HDACs, compared with corresponding normal epithelium. These data show lower expression of class I HDACs, in comparison with those of previous investigations. Toh et al have demonstrated that $26.7-40.0 \%$ of advanced esophageal cancer tissues express HDAC 1 more than does normal mucosa (15), and our data for advanced esophageal cancer tissues showed a similar result. We consider that there were a lower number of cancer tissues in which class I HDACs were overexpressed more than in normal epithelium, because our immunohistochemical study showed that the expression of class I HDACs was equal in many cancer tissues and their corresponding normal epithelia. However, our data also revealed that there were some cases of class I HDAC overexpression in cancer tissues, and HDAC inhibitors may be effective in these class I-overexpressing cancers.

HDACs are thought to play a key role in carcinogenesis. In many studies, overexpression of class I HDACs has been found in cell lines and human cancer tissues; moreover, there have been also some reports that HDAC mRNA expression is a possible prognostic factor $(22,29)$. Therefore, HDAC inhibitors are emerging as a new class of targeted cancer therapeutics. In the current study, class I HDACs were highly expressed in many cancer tissues, but we consider that there are not so many cancer tissues in which class I HDACs are more expressed than the normal epithelium. The activity of class I HDACs is due to histone acetylation status, and HDAC complexes are related to this status (30), therefore, more detailed study, including the expression status of the HDAC complex, is required to predict the effectiveness of HDAC inhibitors. When HDAC inhibitors are used as anti-cancer agents, adjuvant administration should be conbined to avoid side effects, because in many cancers, class I HDACs are overexpressed in cancer and non-cancerous tissues.

\section{References}

1. Grunstein M: Histone acetylation in chromatin structure and transcription. Nature 389: 349-352, 1997.

2. Hassing CA and Schreiber SL: Nuclear histone acetylases and deacetylases and transcriptional regulation: HATs off to HDACs. Curr Opin Chem Biol 1: 300-308, 1997.

3. Struhl K: Histone acetylation and transcriptional regulatory mechanisms. Genes Dev Mar 12: 599-606, 1998.

4. Kuo MH and Allis CD: Roles of histone acetyltransferases and deacetylases in gene regulation. Bioessays 20: 615-626, 1998.

5. Strahl BD and Allis CD: The language of covalent histone modifications. Nature 403: 41-45, 2000.

6. Gregory PD, Wagner K and Horz W: Histone acetylation and chromatin remodeling. Exp Cell Res 265: 195-202, 2001.

7. Thiagalingam S, Cheng KH, Lee HJ, Mineva N, Thiagalingam A and Ponte JF: Histone deacetylases: unique players in shaping the epigenetic histone code. Ann NY Acad Sci 983: 84-100, 2003.

8. De Ruijter AJ, van Gennip AH, Caron HN, Kemp S and van Kuilenburg AB: Histone deacetylases (HDACs): characterization of the classical HDAC family. Biochem J 370: 737-749, 2003.

9. Grozinger CM and Schreiber SL: Deacetylase enzymes: biological functions and the use of small-molecule inhibitors. Chem Biol 9: 3-16, 2002.
10. Park JH, Jung Y, Kim TY, et al: Class I histone deacetylaseselective novel synthetic inhibitors potently inhibit human tumor proliferation. Clin Cancer Res 10: 5271-5281, 2004.

11. Khochbin S, Verdel A, Lemercier C and Seigneurin-Berny D: Functional significance of histone deacetylase diversity. Curr Opin Genet Dev 11: 162-166, 2001.

12. Song J, Noh JH, Lee JH, et al: Increased expression of historic deacetylase 2 is found in human gastric cancer. APMIS 113: 264-268, 2005.

13. Choi JH, Kown HJ, Yoon BI, Kim JH, Han SU, Joo HJ and Kim DY: Expression profile of histone deacetylase 1 in gastric cancer tissue. Jpn J Cancer Res 92: 1300-1304, 2001.

14. Kim JH, Choi YK, Kwon HJ, Yang HK, Choi JH and Kim DY: Downregulation of gelsolin and retinoic acid receptor beta expression in gastric cancer tissues through histone deacetylase 1. J Gastroenterol Hepatol 19: 218-224, 2004.

15. Toh Y, Yamamoto M, Endo K, et al: Histone H4 acetylation and histone deacetylase 1 expression in esophageal squamous carcinoma. Oncol Rep 10: 333-338, 2003.

16. Zhu P, Martin E, Mengwasser J, Schlag P, Janssen KP and Gottlicher M: Induction of HDAC2 expression upon loss of APC in colorectal tumorigenesis. Cancer Cell 5: 455-463, 2004.

17. Giannini R and Cavallini A: Expression analysis of subset of coregulators and three nuclear receptors in human colorectal carcinoma. Anticancer Res 25: 4287-4292, 2005.

18. Patra SK, Patra A and Dahiya R: Histone deacetylase and DNA methyltransferase in human prostate cancer. Biochem Biophys Res Commun 287: 705-713, 2001.

19. Waltregny D, North B, van Mellaert F, De Leval J, Verdin E and Castronovo V: Screening of histone deacetylases (HDAC) expression in human prostate cancer reveals distinct class IHDAC profiles between epithelial and stromal cells. Eur J Histochem 48: 273-290, 2004.

20. Halkidou K, Gaughan L, Cook S, Leung HY, Neal DE and Robson CN: Upregulation and nuclear recruitmen to HDAC1 in hormone refractory prostate cancer. Prostate 59: 177-189, 2004.

21. Krusche CA, Wulfing P, Kersting C, Vloet A, Bocker W, Kiesel L, Beier HM and Alfer J: Histone deacetylase-1 and -3 protein expression in human breast cancer: a tissue microarray analysis. Breast Cancer Res Treat 90: 15-23, 2005.

22. Sasaki H, Moriyama S, Nakashima Y, Kobayashi Y, Kiriyama M, Fukai I, Yamakawa Y and Fujii Y: Histone deacetylase 1 mRNA expression in lung cancer. Lung Cancer 46: 171-178, 2004.

23. Bartling B, Hofmann HS, Boettger T, et al: Comparative application of antibody and gene array for expression profiling in human squamous cell lung carcinoma. Lung Cancer 49: $145-154,2005$.

24. Karagiannis TC and El-Osta A: Modulation of cellular radiation responses by histone deacetylase inhibitors. Oncogene 25: 3885-3893, 2006

25. Maruyama Y, Ono M, Kawahara A, et al: Tumor growth suppression in pancreatic cancer by a putative metastasis suppressor gene Cap43/NDRG1/Drg-1 through modulation of angiogenesis. Cancer Res 66: 6233-6242, 2006.

26. Johnstone RW: Histone-deacetylase inhibitors: novel drugs for the treatment of cancer. Nat Rev Drug Discovery 1: 287-299, 2002.

27. Yang WM, Tsai SC, Wen YD, Fejer G and Seto E: Functional domains of histone deacetylase-3. J Biol Chem 277: 9447-9454, 2002.

28. Van den Wyngaert I, De Vries W, Kremer A, et al: Cloning and characterization of human histone deacetylase 8. FEBS Lett 478: 77-83, 2000.

29. Zhang Z, Tamashita H, Toyama T, et al: Quantitation of HDAC1 mRNA expression in invasive carcinoma of the breast. Breast Cancer Res Treat 94: 11-16, 2005.

30. Inche A and La Thangue NB: Chromatin control and cancer drug discovery: realizing the promise. Drug Discov Today 11: 97-109, 2006. 